\title{
AUTOIMAGEM CORPORAL E PREVALÊNCIA DE SOBREPESO E OBESIDADE EM ESTUDANTES UNIVERSITÁRIOS
}

\section{Body self-image and prevalence of overweight and obesity in university students Autoimagen corporal y prevalencia de sobrepeso y obesidad de estudiantes
universitarios}

\author{
Michelle Alves Vasconcelos Ponte iD \\ Universidade de Trás-os-Montes e Alto Douro - UTAD - Vila Real - Portugal \\ Centro Universitário INTA - UNINTA - Sobral (CE) - Brasil
}

Sandra Celina Fernandes Fonseca (iD

Universidade de Trás-os-Montes e Alto Douro - UTAD - Vila Real - Portugal

Maria Isabel Martins Mourão Carvalhal (DD

Universidade de Trás-os-Montes e Alto Douro - UTAD - Vila Real - Portugal

João José Saraiva da Fonseca (iD

Centro Universitário INTA - UNINTA - Sobral (CE) - Brasil

\section{RESUMO}

Objetivo: Investigar o sobrepeso/obesidade, a autopercepção da imagem corporal de universitários e as associações entre essas variáveis com características sociodemográficas, vinculadas à Universidade e aos comportamentos relacionados à saúde. Métodos: Estudo transversal, exploratório e quantitativo, realizado entre janeiro e fevereiro de 2017, com 324 universitários das áreas da Saúde, Exatas e Humanas. Utilizou-se questionário de Indicadores de Saúde e Qualidade de Vida de Acadêmicos para avaliar características sociodemográficas, vínculo com a universidade e comportamentos relacionados à saúde. Avaliouse a autopercepção da imagem corporal, através da escala de silhuetas, e o estado nutricional, pelo índice de massa corporal. Realizaram-se análises descritivas e regressão Logística Multinominal. Resultados: A prevalência de sobrepeso/obesidade foi de $43,2 \%$ ( $n=141)$ e de insatisfação com a imagem corporal foi de $76,5 \%(n=248)$. As universitárias apresentaram maiores chances de sobrepeso e obesidade (IC 95\%:1,0-2,7; p 0,03). Os estudantes casados (IC 95\%:1,8-5,9; p 0,01), que trabalham (IC 95\%:1,4-3,7; p 0,01), com renda igual ou superior a um salário mínimo (IC 95\%:1,4-4,8; p 0,01), tiveram maior chance de sobrepeso. O estado de saúde regular relacionou-se com maior insatisfação com a imagem corporal (IC 95\%:1,4-5,7; p 0,01). A melhor aptidão física associou-se com menor chance de insatisfação corporal (IC 95\%: 0,06-0,47; p 0,01). Conclusão: Elevada prevalência de sobrepeso/obesidade e de insatisfação com a imagem corporal entre os universitários. Existe associação da prevalência de sobrepeso com faixa etária, sexo, estado civil, trabalho, renda, consumo de carne vermelha, assim como entre a autopercepção da imagem corporal com a autopercepção do estado de saúde, aptidão física e turno de estudo.

Descritores: Obesidade; Avaliação Nutricional; Imagem Corporal; Educação em Saúde; Universidades; Saúde Pública.

\section{ABSTRACT}

Objective: To assess overweight/obesity and self-perception of body image in university students and the associations of these variables with sociodemographic characteristics related to the University and health-related behaviors. Methods: Quantitative exploratory cross-sectional study conducted from January to February 2017 with 324 university students in the fields of Health, Exact and Human sciences. The University Students' Health Indicators and Quality of Life Questionnaire was used to assess sociodemographic characteristics, relationship with the university and health-related behaviors. Body self-image was assessed using the silhouette scale and nutritional status was assessed according to the body mass index. Descriptive analyses and multinominal logistic regression were performed. Results: The prevalence rate of overweight/obesity was $43.2 \%(n=141)$ and the prevalence rate of body image dissatisfaction was $76.5 \%(n=248)$. Female students presented higher chances of being overweight and obese (95\% Cl: 1.0-2.7, p 0.03). Married students (95\% Cl: 1.8-5.9; p 0.01), employed students (95\% Cl: 1.4-3.7; p 0.01) and students with an income equal to or greater than one minimum wage (95\% Cl: 1.4-4.8, $p$ 0.01) were more likely to be overweight. Fair health status was associated with greater body image dissatisfaction (95\% Cl: 1.4-5.7; $p$ 0.01). Better physical fitness was associated with lower chances of body dissatisfaction (95\% Cl: 0.06-0.47; $p$ 0.01). Conclusion: There was a high prevalence of 
overweight/obesity and body image dissatisfaction among university students. Prevalence of overweight was associated with age, sex, marital status, employment, income and red meat consumption whereas self-perception of body image was associated with self-perception of health status, physical fitness and study shift.

Descriptors: Obesity; Nutrition Assessment; Body Image; Health Education; Universities; Public Health.

\section{RESUMEN}

Objetivo: Investigar el sobrepeso/la obesidad, la autopercepción de la imagen corporal de universitarios y las asociaciones entre esas variables y las características sociodemográficas, las vinculadas a la universidad y las conductas relacionadas con la salud. Métodos: Estudio transversal, exploratorio y cuantitativo realizado entre enero y febrero de 2017 con 324 universitarios de las áreas de la Salud, Exactas y Humanas. Se utilizó un cuestionario de Indicadores de Salud y Calidad de Vida de Académicos para evaluar las características sociodemográficas, el vínculo con la universidad y las conductas relacionadas con la salud. Se evaluó la autopercepción de la imagen corporal a través de la escala de siluetas y el estado nutricional por el índice de masa corporal. Se realizaron los análisis descriptivos y de regresión logística multinominal. Resultados: La prevalencia del sobrepeso/ la obesidad ha sido del 43,2\% ( $n=141)$ y de la insatisfacción con la imagen corporal del 76,5\% ( $n=248)$. Las universitarias presentaron mayores probabilidades para el sobrepeso y la obesidad (IC 95\%:1,0-2,7; p 0,03). Los estudiantes casados (IC 95\%:1,8-5,9; $p$ 0,01), con empleo (IC 95\%:1,4-3,7; $p$ 0,01) y renta superior o igual a un sueldo mínimo (IC 95\%:1,4-4,8; $p$ 0,01) tuvieron más probabilidad de sobrepeso. El estado de salud regular se ha relacionado con la mayor insatisfacción con la imagen corporal (IC 95\%:1,4-5,7; $p$ 0,01). La mejor capacidad física se asoció con la baja probabilidad de insatisfacción corporal (IC 95\%: 0,06-0,47; $p$ 0,01). Conclusión: Elevada prevalencia de sobrepeso/obesidad y de insatisfacción con la imagen corporal entre los universitarios. Hay asociación entre la prevalencia de sobrepeso y la franja de edad, el sexo, el estado civil, el trabajo, la renta, el consumo de carne roja así como entre la autopercepción del estado de salud, la capacidad física y el turno de estudio.

Descriptores: Obesidad; Evaluación Nutricional; Imagen Corporal; Educación en Salud; Universidades; Salud Pública.

\section{INTRODUÇÃO}

O Brasil passou por diversas mudanças sociopolíticas, econômicas e culturais, que evidenciaram transformações no modo de vida da população. A ampliação de políticas sociais na área de saúde, educação, trabalho, emprego e assistência social contribuíram para a redução das desigualdades sociais e permitiu que o país crescesse de forma inclusiva. Observou-se, também, a rápida transição demográfica, epidemiológica e nutricional, consequentemente, maior expectativa de vida e redução do número de filhos por mulher. Além disso, é importante destacar as mudanças no padrão de saúde e no consumo alimentar da população brasileira( ${ }^{(1)}$, problemática tratada no presente trabalho.

Atualmente, o Brasil enfrenta problemas de saúde pública, como o sobrepeso e a obesidade. Esses problemas, além de acarretarem riscos à saúde física, podem interferir em como o indivíduo se vê. A imagem corporal é um importante componente do mecanismo de identidade pessoal e esse componente subjetivo corresponde à satisfação e sentimentos relativos à figura mental do corpo, das medidas e dos contornos ${ }^{(2)}$.

Com um olhar ampliado sobre esse contexto e na perspectiva de construir ambientes saudáveis e mudanças de contextos sociais para diminuição de fatores de risco, a Política Nacional de Promoção da Saúde ${ }^{(3)}$ enfatiza a alimentação saudável, que, aliada a práticas corporais e a atividades físicas como ações prioritárias, constituem pilares para o enfrentamento e prevenção a problemas de saúde.

Para a Organização Mundial de Saúde, a obesidade é uma epidemia mundial e está associada a vários processos biopsicossociais, de modo que o ambiente no qual o indivíduo está inserido ganha uma importância de crescente prevalência(4).

O sobrepeso e obesidade em adultos requerem adoção e manutenção de comportamentos de estilo de vida que favoreçam tanto uma alimentação saudável quanto a prática de atividade física. Esses comportamentos são influenciados por muitos fatores, portanto, intervenções incorporando mais de um nível do modelo socioecológico e abordando vários fatores-chave podem ter maior êxito(5).

Assim como em vários países do mundo, o aumento expressivo do sobrepeso e da obesidade deixa o país em alerta e em busca de dados epidemiológicos que ajudem na compreensão de fenômenos que possam subsidiar ações efetivas nesse cenário. É importante destacar que o aumento da obesidade está fortemente ligado ao consumo alimentar e à ausência da prática de atividade física. Seus determinantes são de natureza demográfica, socioeconômica, epidemiológica e cultural, além de questões ambientais, o que torna a obesidade uma doença multifatorial, pois seus determinantes interagem de forma complexa, o que exige que a doença seja tratada considerando-se toda a sua complexidade e a determinação de fatores sociais contextuais. O Estudo de Carga 
Global de Doenças, no Brasil, revela que $58 \%$ dos anos de vida perdidos precocemente estão relacionados às doenças crônicas não transmissíveis ${ }^{(6)}$.

Diante dessa demanda, que interliga conceitos educacionais e de saúde a partir de uma visão ampliada do indivíduo que adentra o ensino superior, o processo formativo é compreendido não apenas como preparo no domínio de conceitos teóricos e técnicas eficazes, mas também como a dimensão humana do processo de ensinoaprendizagem.

Portanto, o objetivo do presente estudo foi investigar o sobrepeso/obesidade, a autopercepção da imagem corporal de universitários e as associações entre essas variáveis com características sociodemográficas vinculadas à universidade e à comportamentos relacionados à saúde.

\section{MÉTODOS}

Trata-se de um estudo transversal, exploratório, com abordagem quantitativa, com recolha de dados nos meses de janeiro e fevereiro de 2017, que faz parte de uma pesquisa, tese de doutorado em Ciências da Educação da Universidade Trás-os-Montes e Alto Douro - UTAD/Portugal, sobre o processo de adaptação ao ensino superior: fatores associados ao rendimento acadêmico e à qualidade de vida em estudantes universitários, realizado em uma instituição de ensino superior da região Noroeste do Ceará, Brasil.

A população do estudo foi constituída por 420 estudantes universitários, regularmente matriculados no $2^{\circ}$ semestre letivo e no último ano letivo dos seguintes cursos: Arquitetura e Urbanismo, Educação Física, Enfermagem, Farmácia, Fisioterapia, Jornalismo, Medicina Veterinária, Nutrição, Pedagogia e Serviço Social.

Utilizou-se o critério de proporcionalidade da amostra para a definição do tamanho amostral, considerando-se a relação entre o número de alunos em cada área que atendiam aos critérios elegíveis. Dessa distribuição populacional, retirou-se uma proporção de cada curso, a fim de garantir uma amostra representativa.

A amostragem foi determinada de acordo com um plano de seleção proporcional às áreas e calculada com base no erro amostral de $5 \%$ e um nível de confiança de $95 \%$. Foram acrescidos $20 \%$ para possíveis perdas e recusas e $15 \%$ para o controle de fatores de confusão em estudos de associação, totalizando uma amostra de 324 estudantes, assim distribuídos: curso de Arquitetura e Urbanismo (10,2\%; n=33), Educação Física (12,7\%; n=41), Enfermagem $(11,4 \% ; n=37)$, Farmácia $(11,4 \% ; n=37)$, Fisioterapia $(7,7 \% ; n=25)$, Jornalismo $(9,0 \% ; n=29)$, Medicina Veterinária $(10,8 \% ; n=35)$, Nutrição $(9,3 \% ; n=30)$, Pedagogia $(8,6 \% ; n=28)$ e Serviço Social $(9,0 \% ; n=29)$.

Os critérios de exclusão foram: estudantes que não estavam cursando o semestre regular, mas apenas disciplinas isoladas, alunos com ano de entrada na universidade divergente da turma estudada e alunos dos cursos de graduação a distância.

Utilizou-se como instrumento de coleta o ISAQ-A (Indicadores de Saúde e Qualidade de Vida de Acadêmicos) ${ }^{(7)}$, pois este questionário apresenta características satisfatórias de aplicabilidade, validade e reprodutibilidade para 0 uso em pesquisas com estudantes universitários brasileiros. O instrumento utilizado é composto por sete seções: (1) informações sobre o curso, (2) indicadores sociodemográficos, (3) indicadores do estilo de vida e saúde, (4) hábitos alimentares e controle do peso corporal, (5) atividades físicas e opções de lazer, (6) comportamentos preventivos e (7) indicadores do ambiente e das condições de aprendizagem.

É importante destacar que a aplicação do ISAQ-A ocorreu por meio de uma equipe de docentes previamente treinados para a recepção dos participantes, preenchimento dos dados gerais, verificação das medidas antropométricas. Além disso, a adesão dos estudantes ocorreu de forma voluntária.

Por se tratar de uma pesquisa com foco na qualidade de vida dos estudantes, houve uma preocupação com a acolhida deles, priorizando-se a ética e a humanização. Ofereceram-se condições de conforto aos alunos, espaço físico adequado, tempo suficiente para responder ao questionário e espaço reservado para aferição das medidas.

As variáveis do estudo dividiram-se em dependentes e exploratórias, e os desfechos das variáveis dependentes foram: avaliação nutricional (eutróficos/ baixo peso e sobrepeso/ obesidade) e autopercepção da imagem corporal (satisfeito e insatisfeito).

Seguem os passos percorridos para escolha das variáveis desfechos. Na classificação por índice de massa corporal (IMC) dos estudantes das amostras femininas e masculinas, consideraram-se os dados reais das medidas antropométricas: peso e altura. O IMC foi obtido a partir da massa corporal (MC, em quilogramas, $\mathrm{kg}$ ) e estatura (E, em metros, $\mathrm{m}$ ). A classificação do IMC dos indivíduos com idade igual ou superior a 18 anos foi realizada da seguinte maneira: baixo peso - menor 18,5 kg/m²; eutrófico (peso normal) - entre 18,5 e 24,9 kg/m²; sobrepeso - entre 25,0 e $29,9 \mathrm{~kg} / \mathrm{m}^{2}$; obesidade - maior ou igual $30,0 \mathrm{~kg} / \mathrm{m}^{2(7)}$. 
Utilizou-se uma balança eletrônica calibrada (Kratos-Cas ${ }^{\circledR}$, Brasil) para a tomada das medidas de peso, e os sujeitos foram pesados sem calçados, sem peças de vestuário nem objetos pesados. Para a medida da estatura, utilizou-se um antropômetro portátil (Kratos-Cas ${ }^{\circledR}$, Brasil).

Para avaliação do estado nutricional, agruparam-se os estudantes com baixo peso na categoria eutrófico (referência), devido à baixa frequência para essa categoria, e agrupou-se os estudantes com sobrepeso e obesidade na categoria sobrepeso.

Em relação à autopercepção da imagem corporal, utilizou-se a escala de figuras de silhuetas( ${ }^{(8)}$, que consiste num conjunto de nove silhuetas de cada gênero, apresentadas em figuras individuais, com variações progressivas na escala de medida (da figura mais magra a mais larga), com IMC médio variando entre 17,5 e $37,5 \mathrm{~kg} / \mathrm{m}^{2}$. Cada estudante foi convidado a escolher a figura que melhor o representasse e a que gostaria de ter ou que julga ser 0 ideal. A satisfação ou insatisfação com a imagem corporal é avaliada conforme as discrepâncias entre as figuras selecionadas (Figura 1). Dessa forma, os desfechos foram agrupados em duas categorias: Satisfeito com a imagem corporal e Insatisfeito com a imagem corporal.

As figuras da silhueta corporal(8) foram validadas com população de brasileiros, adultos e universitários, utilizadas como referência no questionário deste estudo.

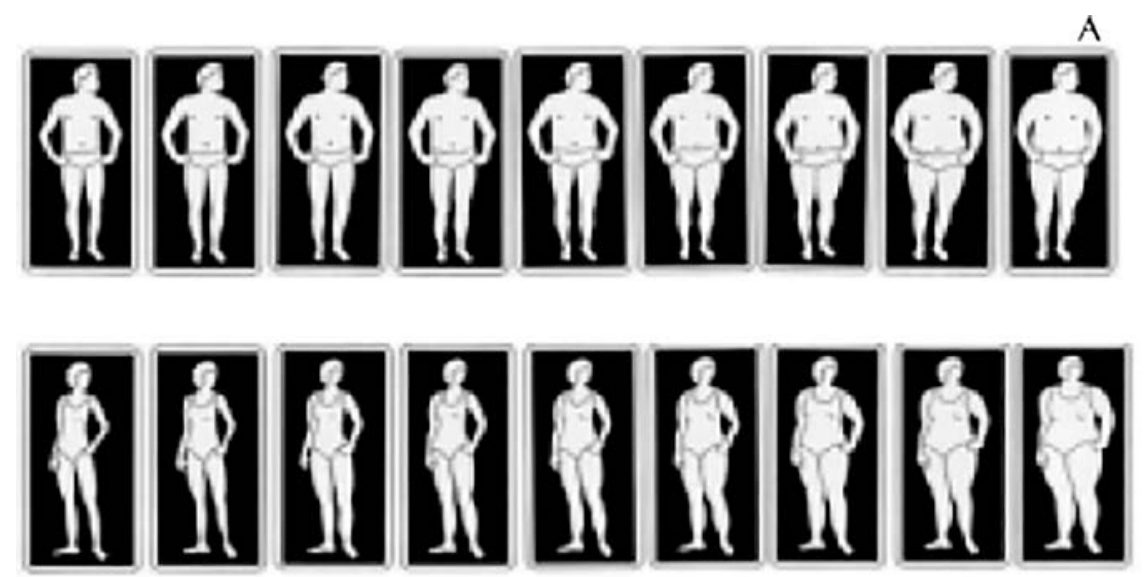

Figura 1 - Escala de Figura de Silhuetas de cada sexo, apresentam IMC médio variando entre 17,5 a $37,5 \mathrm{~kg} / \mathrm{m}^{2(8)}$.

As variáveis exploratórias foram divididas em (1) características sociodemográficas: sexo, faixa etária, estado civil, trabalho, carga horária e renda; (2) características de vínculo com a universidade: áreas, tempo de exposição à universidade, turno de estudo e oportunidade de ensino, pesquisa e extensão; (3) comportamentos relacionados à saúde: atividade física no lazer, hábitos alimentares (consumo semanal de frutas, verduras e carne vermelha; frango com pele; salgadinhos; refrigerantes; refeições em restaurantes universitários) e comportamentos saudáveis.

Realizou-se a análise dos dados por meio do exame descritivo e inferencial da amostra de tabelas de frequência e da análise de média, desvio padrão, mínimo e máximo. Utilizou-se o programa SPSS $20.0^{\circledR}$ (IBM, USA) para verificar associações nos testes, como teste de Qui-quadrado e razões de prevalência.

Para realização do estudo, respeitaram-se os princípios éticos da Resolução $466 / 12^{(9)}$ do Conselho Nacional de Saúde, que versa sobre as pesquisas envolvendo seres humanos. O projeto foi enviado ao Comitê de Ética em Pesquisa do Brasil e obteve aprovação sob Parecer $n^{\circ}$. 1.910.729.

\section{RESULTADOS}

O presente estudo foi formado pelos seguintes participantes: 324 estudantes universitários, com perfil descritivo em maioria do sexo feminino $(67,9 \%$; $n=220)$, solteiros $(81,2 \% ; n=263)$, com faixa etária entre 20 a 29 anos $(68,5 \%$; $n=222)$, residentes na cidade onde se localiza a instituição de ensino $(65,4 \% ; n=212)$ e moram com seus pais $59,9 \%$ $(n=194)$. Além disso, utilizam moto ou carro próprio $(41,7 \% ; n=135)$ como meio de locomoção para a instituição de ensino.

Observou-se que a escolaridade das mães foi maior. Destas, 33\% $(n=107)$ concluíram o ensino médio, enquanto que 41,4\% ( $n=134)$ dos pais têm apenas o ensino fundamental. Com relação ao emprego e renda, 52,5\% ( $n=170)$ dos estudantes não trabalham e 56,5\% ( $n=183)$ não têm renda própria. Dos que afirmaram trabalhar ou estagiar, $42,2 \%(n=65)$ têm entre 20 a 40 horas semanais destinadas a essas atividades, sendo a renda de $2,5 \%(n=8)$ menor que 1 (um) salário mínimo. 
Na Tabela I, é possível verificar a prevalência de sobrepeso e obesidade em 43,2\% ( $n=141)$ dos estudantes; $50 \%(n=162)$ dos estudantes estão insatisfeitos com sua imagem e desejam diminuir seu peso; e $26,5 \%(n=86)$ estão insatisfeitos com sua imagem e desejam aumentar seu peso.

Tabela I - Distribuição dos estudantes universitários segundo avaliação nutricional e autopercepção da imagem corporal. Sobral, Ceará, Brasil, 2017.

\begin{tabular}{lcc}
\hline Características & $\mathbf{n}$ & $\%$ \\
\hline Classificação do IMC & 7 & \\
Baixo peso & 176 & 2,2 \\
Eutrófico & 106 & 54,3 \\
Sobrepeso & 35 & 32,7 \\
Obesidade & 1 & 10,5 \\
Não informado & & 0,3 \\
Classificação da imagem corporal & 75 & 23,1 \\
Satisfeito & 86 & 26,5 \\
Insatisfeito/deseja aumentar o peso & 162 & 50,0 \\
Insatisfeito/deseja diminuir o peso & 1 & 0,3 \\
Não informou &
\end{tabular}

IMC: Índice de massa corporal.

Não houve associação entre a prevalência de sobrepeso e as áreas de conhecimento (Exatas, Humanas e da Saúde) cursadas pelos estudantes. Também não foi possível observar associação significante entre o sobrepeso e o tempo dos estudantes na universidade.

Nota-se, na Tabela II, que houve associação entre sexo e prevalência de sobrepeso. Estudantes do sexo feminino tiveram 1,7 vezes mais chance de ter sobrepeso/obesidade, com associação entre a faixa etária e prevalência de sobrepeso. Tomando como referência a faixa etária 30 anos ou mais, o indivíduo com idade menor de 20 anos tem $77 \%$ menos chance de ter sobrepeso, e o indivíduo com idade entre 20 e 29 anos tem $65 \%$ menos chance de ter sobrepeso.

Quanto ao estado civil, os estudantes casados/separados/viúvos tiveram 3,3 vezes mais chance de apresentar sobrepeso do que o grupo dos solteiros. De acordo com o trabalho e a renda, tendo como referência o grupo que não trabalha, a renda mensal apresentou associação com a prevalência de sobrepeso. O grupo que recebe 1 salário mínimo ou mais apresenta 2,6 vezes mais chance de ter obesidade.

Tabela II - Comparação entre prevalência de sobrepeso e características sociodemográficas dos universitários. Sobral, Ceará, Brasil, 2017.

\begin{tabular}{|c|c|c|c|c|c|c|c|}
\hline \multirow[t]{2}{*}{ Características } & \multicolumn{2}{|c|}{$\begin{array}{l}\text { Baixo pesol } \\
\text { Eutrófico }\end{array}$} & \multicolumn{2}{|c|}{$\begin{array}{l}\text { Sobrepesol } \\
\text { obesidade }\end{array}$} & \multirow[t]{2}{*}{$\mathbf{p}$} & \multirow[t]{2}{*}{ OR } & \multirow[t]{2}{*}{ IC $95 \%$} \\
\hline & $\mathbf{n}$ & $\%$ & $\mathbf{n}$ & $\%$ & & & \\
\hline \multicolumn{8}{|l|}{ Sexo } \\
\hline Masculino & 133 & 60,7 & 86 & 39,3 & \multirow[t]{2}{*}{$0,03^{*}$} & 1,0 & \\
\hline Feminino & 50 & 48,1 & 54 & 51,9 & & 1,7 & {$[1,0-2,7]$} \\
\hline \multicolumn{8}{|l|}{ Faixa etária } \\
\hline$<20$ anos & 42 & 67,7 & 20 & 32,3 & \multirow[t]{3}{*}{$<0,01^{*}$} & 0,23 & {$[0,10-0,54]$} \\
\hline De 20 a 29 anos & 128 & 57,9 & 93 & 42,1 & & 0,35 & {$[0,17-0,71]$} \\
\hline 30 anos ou mais & 13 & 32,5 & 27 & 67,5 & & 1,00 & - \\
\hline \multicolumn{8}{|l|}{ Estado civil } \\
\hline Solteiro & 163 & 62,0 & 100 & 38,0 & \multirow[t]{2}{*}{$<0,01^{*}$} & 1,0 & - \\
\hline Casado/Divorciado/Viúvo & 20 & 33,3 & 40 & 66,7 & & 3,3 & {$[1,8-5,9]$} \\
\hline \multicolumn{8}{|l|}{ Trabalho } \\
\hline Não trabalha & 111 & 65,3 & 59 & 34,7 & \multirow[t]{3}{*}{$0,01^{*}$} & 1,0 & - \\
\hline Estágio & 22 & 51,2 & 21 & 48,8 & & 1,8 & {$[0,9-3,5]$} \\
\hline Trabalha com/sem salário & 47 & 46,1 & 55 & 53,9 & & 2,2 & {$[1,4-3,7]$} \\
\hline \multicolumn{8}{|l|}{ Carga horária } \\
\hline Até 20 horas/semana & 33 & 51,6 & 31 & 48,4 & \multirow[t]{3}{*}{0,39} & - & \multirow[t]{3}{*}{-} \\
\hline 20 | 40 horas/semana & 26 & 40,0 & 39 & 60,0 & & & \\
\hline Mais de 40 horas/semana & 11 & 50,0 & 11 & 50,0 & & & \\
\hline \multicolumn{8}{|l|}{ Renda } \\
\hline Sem renda & 115 & 62,8 & 68 & 37,2 & \multirow[t]{3}{*}{$0,01^{*}$} & 1,0 & \\
\hline$<1 \mathrm{SM}$ & 44 & 53,7 & 38 & 46,3 & & 1,5 & {$[0,9-2,5]$} \\
\hline $1 \mathrm{SM}$ ou mais & 22 & 39,3 & 34 & 60,7 & & 2,6 & {$[1,4-4,8]$} \\
\hline
\end{tabular}

SM: Salário Mínimo; Teste de Qui-quadrado; Análise de Regressão Logística; * ${ }^{p}<0,05$; OR: Odds ratio; IC: intervalo de confiança 
Quanto ao consumo semanal de alimentos, não houve associação significativa entre a prevalência de sobrepeso, o consumo de frutas e o consumo de verduras por mais de 3 dias/semana.

A Tabela III demonstra que a média de consumo de carne vermelha no grupo sobrepeso/obesidade foi de 3,5 dias/ semana, enquanto que no grupo dos eutróficos foi de 3,0 dias/semana. À medida que o consumo de carne vermelha aumenta, a chance de ter sobrepeso/ obesidade é 1,15 vezes maior. Não houve associação entre prevalência de sobrepeso e o consumo de salgadinhos e refrigerantes neste grupo.

Tabela III - Comparação entre prevalência de sobrepeso e consumo semanal de alimentos. Sobral, Ceará, Brasil, 2017.

\begin{tabular}{|c|c|c|c|c|c|c|c|}
\hline \multirow[t]{2}{*}{ Características } & \multicolumn{2}{|c|}{$\begin{array}{c}\text { Baixo pesol } \\
\text { eutrófico }\end{array}$} & \multicolumn{2}{|c|}{$\begin{array}{c}\text { Sobrepeso/ } \\
\text { obesidade }\end{array}$} & \multirow[t]{2}{*}{$\mathbf{p}$} & \multirow[t]{2}{*}{ OR } & \multirow[t]{2}{*}{ IC $95 \%$} \\
\hline & Média & DP & Média & DP & & & \\
\hline \multicolumn{8}{|c|}{ Consumo semanal de alimentos } \\
\hline Frutas & 3,3 & 2,1 & 3,4 & 2,1 & 0,82 & - & - \\
\hline Verduras & 3,1 & 2,3 & 3,0 & 2,3 & 0,70 & - & - \\
\hline Carne vermelha & 3,0 & 1,7 & 3,5 & 1,9 & $0,03^{*}$ & 1,15 & {$[1,01-1,30]$} \\
\hline Frango com pele & 2,1 & 2,0 & 2,5 & 2,2 & 0,05 & - & - \\
\hline Salgadinhos & 2,6 & 2,0 & 2,7 & 2,0 & 0,64 & - & - \\
\hline Refrigerantes & 2,5 & 2,2 & 2,5 & 2,3 & 0,94 & - & - \\
\hline \multicolumn{8}{|l|}{ Refeições no RU } \\
\hline Café da manhã & 0,5 & 1,3 & 0,3 & 1,0 & 0,27 & - & - \\
\hline Almoço & 0,1 & 0,3 & 0,2 & 0,8 & 0,08 & - & - \\
\hline Jantar & 0,4 & 1,1 & 0,4 & 1,1 & 0,98 & - & - \\
\hline
\end{tabular}

Teste t-Student; Análise de Regressão Logística; * $\mathrm{p}<0,05$; RU: restaurante universitário; OR: Odds ratio; DP: desvio padrão; IC: intervalo de confiança

Consoante observado sobre a autoimagem corporal (Tabela IV), os resultados apontaram que o estudante que estuda em tempo integral (manhã e tarde), como no curso de Medicina Veterinária, tem 2,9 vezes mais chance de estar insatisfeito com a imagem; enquanto o estudante do turno noturno tem 2 vezes mais chance de estar insatisfeito com a imagem corporal. As demais características não apresentaram associação com autopercepção da imagem corporal.

Tabela IV - Comparação entre autopercepção da imagem corporal e vínculo com a universidade. Sobral, Ceará, Brasil, 2017.

\begin{tabular}{|c|c|c|c|c|c|c|c|}
\hline \multirow{2}{*}{ Características } & \multicolumn{2}{|c|}{ Satisfeito } & \multicolumn{2}{|c|}{ Insatisfeito } & \multirow{2}{*}{ p } & \multirow{2}{*}{ OR } & \multirow{2}{*}{ IC $95 \%$} \\
\hline & $n$ & $\%$ & $n$ & $\%$ & & & \\
\hline \multicolumn{8}{|l|}{ Área } \\
\hline Exatas & 10 & 30,3 & 23 & 69,7 & 0,48 & - & - \\
\hline Humanas & 24 & 24,7 & 73 & 75,3 & & & \\
\hline Saúde & 41 & 21,2 & 152 & 78,8 & & & \\
\hline \multicolumn{8}{|c|}{ Anos de exposição à universidade } \\
\hline$<1$ ano & 30 & 20,4 & 117 & 79,6 & 0,32 & - & - \\
\hline 3 anos & 24 & 30,8 & 54 & 69,2 & & & \\
\hline 4 anos & 15 & 22,7 & 51 & 77,3 & & & \\
\hline 5 anos & 6 & 18,8 & 26 & 81,2 & & & \\
\hline \multicolumn{8}{|l|}{ Situação } \\
\hline Concluinte & 45 & 25,6 & 131 & 74,4 & 0,29 & & \\
\hline Ingressante & 30 & 20,4 & 117 & 79,6 & & & \\
\hline \multicolumn{8}{|l|}{ Turno } \\
\hline Manhã & 42 & 31,6 & 91 & 68,4 & $0,02^{*}$ & 1 & \\
\hline Tarde & 6 & 16,2 & 31 & 83,8 & & 2,4 & {$[0,9-6,1]$} \\
\hline Integral & 5 & 13,5 & 32 & 86,5 & & 2,9 & {$[1,1-8,1]$} \\
\hline Noturno & 22 & 19,0 & 94 & 81,0 & & 2,0 & {$[1,1-3,6]$} \\
\hline
\end{tabular}

Teste t-Student; Análise de Regressão Logística; * ${ }^{\circ}<0,05$; n: Número absoluto; OR: Odds ratio; IC: intervalo de confiança 
Em relação ao grupo de autopercepção positiva, os estudantes que classificaram seu estado de saúde como regular têm 2,8 vezes mais chance de estarem insatisfeitos com a imagem corporal, como descrito na Tabela V. Quando observada a relação entre a autopercepção da imagem corporal e a aptidão física, tendo como referência o indivíduo que considera a sua aptidão física pior que os semelhantes, o indivíduo que tem aptidão física melhor tem $83 \%$ menos chance de estar insatisfeito com a imagem corporal; enquanto o que tem aptidão física semelhante tem $76 \%$ menos chance de estar insatisfeito com a imagem corporal.

Tabela $V$ - Comparação entre autopercepção da imagem corporal e percepção do estado de saúde e aptidão física comparado aos seus semelhantes. Sobral, Ceará, Brasil, 2017.

\begin{tabular}{|c|c|c|c|c|c|c|c|}
\hline \multirow{2}{*}{ Percepção } & \multicolumn{2}{|c|}{ Satisfeito } & \multicolumn{2}{|c|}{ Insatisfeito } & \multirow{2}{*}{ p } & \multirow{2}{*}{ OR } & \multirow{2}{*}{ IC $95 \%$} \\
\hline & $\mathrm{n}$ & $\%$ & $\mathrm{n}$ & $\%$ & & & \\
\hline Positiva & 62 & 28,8 & 153 & 71,2 & $<0,01^{*}$ & - & - \\
\hline Regular & 11 & 12,5 & 77 & 87,5 & & 2,8 & {$[1,4-5,7]$} \\
\hline Negativa & 2 & 10,0 & 18 & 90,0 & & 3,6 & {$[0,8-16,2]$} \\
\hline \multicolumn{8}{|l|}{ Aptidão Física } \\
\hline Pior & 6 & 8,2 & 67 & 91,8 & $<0,01^{*}$ & 1,00 & \\
\hline Melhor & 17 & 34,7 & 32 & 65,3 & & 0,17 & {$[0,06-0,47]$} \\
\hline Semelhante & 34 & 27,4 & 90 & 72,6 & & 0,24 & {$[0,09-0,60]$} \\
\hline Não sabe & 18 & 23,4 & 59 & 76,6 & & 0,29 & {$[0,11-0,79]$} \\
\hline \multicolumn{8}{|c|}{ Prática de atividade física de lazer } \\
\hline Ativo & 49 & 27,5 & 129 & 72,5 & 0,06 & - & - \\
\hline Inativo & 26 & 18,2 & 117 & 81,8 & & & \\
\hline
\end{tabular}

Teste t-Student; Análise de Regressão Logística; * $p$ <0,05; OR: Odds ratio; IC: intervalo de confiança

\section{DISCUSSÃO}

No presente estudo constatou-se a prevalência de $76,5 \%$ de insatisfação com a imagem corporal. A maioria dos estudantes insatisfeitos com sua imagem corporal tem uma visão negativa de seu estado de saúde. A percepção da imagem corporal também está associada à avaliação de sua aptidão física com seus semelhantes, uma vez que os estudantes que se avaliam com melhor aptidão física têm menor chance de imagem corporal negativa.

Levando em consideração as construções imagéticas no mundo contemporâneo, infere-se que tais resultados estão relacionados com as exigências do estereótipo social feminino. Em pesquisa nacional realizada entre escolares houve associação entre a percepção da imagem corporal e os comportamentos extremos para controle de peso, com maior força para meninas que se sentiam muito gordas. Tais resultados permitem inferir que a percepção da imagem corporal pode ter maior influência na adoção dos comportamentos extremos ${ }^{(10)}$.

Ao investigar os consumos e estilos de vida, um estudo com estudantes da Universidade de Lisboa ${ }^{(11)}$ demonstra que a forma física pode ser vista a partir de uma configuração corporal subjetiva, produzida por si próprio, pelos outros e por processos de avaliação e de reconhecimento da adequação do indivíduo a um "estilo de vida saudável". Esse olhar do estudante sobre o seu corpo, sua silhueta e as sensações que este lhe devolve, em comparação social com outros corpos, despertam mecanismos subjetivos de avaliação corporal, os quais têm efeitos na autoestima do indivíduo, no bem-estar individual e social, possibilitando estratégias de manutenção ou modificação corporal no sentido de um corpo socialmente reconhecido como "saudável".

O grau de excelência na avaliação da forma física varia ainda com o sexo, sendo as estudantes mais exigentes do que os rapazes na sua avaliação de excelente ${ }^{(11)}$. Esses dados confirmam os achados deste estudo: as estudantes estão mais insatisfeitas com sua imagem corporal e, quanto maior a faixa etária, a avaliação negativa aumenta em ambos os sexos.

A felicidade, enquanto autopercepção de satisfação - que contribui na capacidade de decidir como aproveitar a vida, como ser um sujeito ativo na construção de projetos e intervenções para superar dificuldades individuais e coletivas, e no reconhecimento de potencialidades - é um dos valores fundamentais para que a promoção da Saúde seja efetivada ${ }^{(3)}$.

Nesse sentido, atividades, projetos ou componentes curriculares que favoreçam a reflexão dos estudantes sobre si, sobre sua adaptação na educação superior e que também potencializem condições de enfrentamento de adversidades da vida - seja acadêmica seja pessoal -, devem fazer parte do cotidiano universitário. 
As instituições de ensino superior configuram-se como espaço ideal para debates e discussões em torno das temáticas sobre qualidade de vida, obesidade, hábitos alimentares e apreciações sobre saúde. Além disso, ambientes saudáveis são privilegiados para potencializar a promoção e a prevenção, como sugere a Política Nacional de Promoção da Saúde ${ }^{(3)}$.

A escala de silhuetas representou um instrumento bastante eficaz para avaliar o grau de insatisfação com o peso e as dimensões corporais na avaliação do componente perceptivo da imagem corporal, e contribuiu, também, para a compreensão de como o estudante idealiza sua imagem e como vê sua imagem objetiva( ${ }^{(8)}$.

O sobrepeso foi prevalente em $43,2 \%$ dos estudantes, e as mulheres universitárias apresentam maiores chances de sobrepeso e obesidade que os homens. Dados mostram que o Brasil vem enfrentando o aumento progressivo do sobrepeso e obesidade. Sendo um dos principais fatores de risco para doenças crônicas não transmissíveis e um problema de saúde pública, o excesso de peso acomete 1 (um) em cada 2 (dois) adultos e (1) uma em cada (3) três crianças brasileiras ${ }^{(1)}$.

Em adultos, acima de 18 (dezoito) anos, residentes em Fortaleza, Ceará, a frequência de obesidade verificada pela Vigilância de Fatores de Risco e Proteção para Doenças Crônicas por Inquérito Telefônico (VIGITEL) foi de $19,2 \%{ }^{(12)}$.

Quanto à associação significativa entre sobrepeso/obesidade no sexo feminino, outro estudo, também com população universitária, evidenciou diferenças dos resultados encontrados neste estudo, uma vez que as maiores prevalências de sobrepeso/obesidade foram encontradas nos estudantes do sexo masculino. É importante destacar que este estudo foi um inquérito transversal, repetido no período de 2 (dois) anos, de 2010 a 2012, com a participação de mais de 1200 estudantes de uma universidade pública e com a aplicação do mesmo instrumento de coleta de $\operatorname{dados}^{(13)}$.

Consoante observado, os dados confirmam a tendência da população brasileira descrita na Pesquisa Nacional de Saúde no ano de 2013. No que diz respeito à análise do excesso de peso da população adulta, estratificada por grupos de idade, estimou-se que mais da metade da população apresentou excesso de peso (56,9\%), ou seja, cerca de 82 milhões de brasileiros apresentaram o IMC igual ou maior do que $25 \mathrm{~kg} / \mathrm{m} 2$, indicando uma prevalência maior de excesso de peso no sexo feminino, $58,2 \%{ }^{(6)}$.

O comportamento das prevalências de excesso de peso e de obesidade ao longo de três pesquisas (2003, 2008 e 2013), evidenciou um comportamento inverso, aumentando continuamente tanto para os homens quanto para as mulheres. Para os homens, a prevalência de excesso de peso aumentou de 42,4\%, em 2002-2003, para 57,3\%, em 2013, já a obesidade aumentou de $9,3 \%$ para $17,5 \%$. No caso das mulheres, esse aumento foi mais acentuado, passando de $42,1 \%$, em 2002-2003, para 59,8\%, em 2013, já a obesidade passou de $14,0 \%$ para $25,2 \%{ }^{(6)}$.

Confirmando os resultados do presente estudo, em que estudantes menores que 20 anos têm menos chances de sobrepeso e obesidade, um estudo com universitários da Região Sul do Brasil(14) demonstrou uma associação entre o excesso do peso corporal e a faixa etária. A magnitude da associação nos estudantes com 20 anos ou mais de idade foram 2 (duas) vezes maiores que seus pares mais jovens, e os resultados de maior prevalência de sobrepeso na maior faixa etária foram encontrados em outros estudos ${ }^{(1,6,13)}$.

No que diz respeito às demais variáveis sociodemográficas encontradas na presente pesquisa, os estudantes casados/separados/viúvos, com trabalho e renda maior que 1 (um) salário mínimo, têm maior chance de apresentarem sobrepeso. Esses resultados também foram encontrados em estudo com universitários de uma instituição pública no Piauí, em que o risco de os estudantes desenvolverem excesso de peso foi maior nos níveis socioeconômicos médio e alto(15).

A ampliação do olhar sobre o fenômeno do crescimento do sobrepeso entre os universitários é necessária, uma vez que a identificação dos fatores associados a comportamentos de risco pode direcionar políticas públicas e o desenvolvimento de programas que favoreçam o ambiente universitário como promotor de culturas saudáveis.

A vigilância, monitoramento e avaliação, enquanto uso de múltiplas abordagens na geração e análise de informações sobre as condições de saúde, para subsidiar decisões, intervenções e implantar políticas públicas de promoção da saúde, compõe um dos eixos da Política Nacional de Promoção da Saúde ${ }^{(3)}$. Nesse sentido, percebe-se a importância de indicadores que norteiem a implantação de projetos e programas voltados à população universitária.

Os estereótipos negativos, por vezes presentes em pessoas com sobrepeso, influenciam de maneira negativa a adesão a programas, de modo a desmotivar esses indivíduos, resultando no reluto ao tratamento. Portanto, estratégias educativas que enfatizem a complexa etiologia da obesidade podem reduzir os estereótipos de peso entre os estudantes, devendo integrar os currículos e os programas ${ }^{(16)}$. 
Através de um chamamento público, o Ministério da Saúde incentiva universidades públicas e privadas a desenvolverem projetos com o objetivo de prevenir e controlar a obesidade no âmbito do Sistema Único de Saúde, também como forma de qualificar a assistência, o cuidado e o desenvolvimento de pesquisas ${ }^{(17)}$. Entretanto, é válido refletir que a população universitária precisa ser visualizada não somente na posição de produzir conhecimentos e boas práticas para, sobretudo, que seja alcançada por essas ações.

Quando se estudou a prevalência de sobrepeso e a associação entre as áreas cursadas (Saúde, Humanas e Exatas), não se observou nenhuma associação significativa na atual investigação. $O$ fato dos estudantes dos cursos de Saúde, estudarem comportamentos saudáveis e outros conhecimentos transversais sobre a temática não os diferenciou dos demais.

Na Região Sul do estado da Bahia(13), um estudo evidenciou, em estudantes (sexo masculino) no segundo ano de exposição à universidade e em estudantes (sexo feminino) no quarto ano ou mais de exposição à universidade, menores estimativas de associação com a obesidade. Os efeitos da associação foram menores para os estudantes das áreas de Ciências Exatas, da Terra e Humanas. Já com universitários de outro estado, as prevalências de excesso de peso corporal aumentaram juntamente com o tempo de exposição à universidade ${ }^{(18)}$.

Quanto ao consumo de alimentos, considerado marcador de padrões saudáveis, avaliou-se a frequência de frutas e verduras e os padrões não saudáveis de alimentação, isto é, o consumo de carne vermelha, refrigerantes e salgadinhos pelos estudantes do presente estudo. E não se observou diferença entre os grupos de desfecho em relação ao consumo de frutas e verduras, salgadinhos e refrigerantes. Todavia, os estudantes com sobrepeso/ obesidade apresentaram maior prevalência no consumo de carne vermelha e frango com pele, visto que aumentaram as chances de obesidade quando aumentou o consumo de carne vermelha.

Consoante uma pesquisa com universitários ${ }^{(13)}$, estudantes do sexo masculino que referiram o consumo de frutas até 4 dias/semana estavam menos propensos a obesidade, no entanto os estudantes universitários que reportaram o consumo de hortaliças até 4 dias/semana estavam mais propensos à obesidade, assim como em relação ao consumo de carne com gordura visível sobre o sobrepeso. Para as mulheres, aquelas que consumiram refrigerantes em cinco ou mais dias por semana estavam mais associadas à obesidade. Em contrapartida, as que relataram consumir salgados estavam menos propensas a obesidade.

Notou-se que a ingestão de frutas e de verduras dos estudantes analisados no atual estudo está abaixo da recomendada pela Organização Mundial de Saúde, que é de 400 gramas diários em cinco ou mais dias da semana $^{(19)}$. Essa problemática vai ao encontro do inquérito nacional, no qual apenas $24,1 \%$ dos brasileiros ingerem a quantidade de frutas e hortaliças recomendada, e $29,4 \%$ da população ainda consome carne com excesso de gordura. Os números indicam também que o brasileiro tem diminuído a ingestão de refrigerante, com queda de cerca $20 \%$ nos últimos seis anos. Entretanto mais de $20 \%$ da população ainda ingere esse tipo bebida cinco vezes ou mais na semana ${ }^{(11)}$.

A ingestão de alimentos saudáveis está abaixo do recomendado. O fato de estudantes da área de Saúde entrevistados na atual pesquisa não apresentarem diferenças relacionadas ao sobrepeso, aos hábitos alimentares, leva a reflexão sobre como o conhecimento de prevenção e promoção da saúde trabalhado durante seus cursos, por si só, não garante a adoção de hábitos saudáveis.

O acelerado crescimento do excesso de peso em todas as faixas etárias e de renda deixa clara a necessidade de medidas de controle e prevenção do ganho de peso. Caso as ações não sejam implementadas, estima-se que, em vinte anos, cerca de $70 \%$ dos brasileiros estarão com excesso de peso. O enfrentamento requer ações nos diversos setores, desde a produção à comercialização final dos alimentos, e com a garantia de ambientes que propiciem a mudança de conduta dos indivíduos e da sociedade(1).

Esta pesquisa corrobora as necessidades apontadas em estudo com adolescentes escolares ${ }^{(20)}$ sobre: a importância de orientação e estímulo às escolhas alimentares saudáveis; disponibilização de prática de exercícios físicos no espaço escolar; disponibilização de opções de alimentos saudáveis nas cantinas; orientação para redução e prevenção de sobrepeso. No âmbito da universidade, apontamos a importância dos restaurantes universitários, dos núcleos de apoio aos discentes (NAPs), dos projetos de extensão voltados para a promoção da saúde, de espaços e ações que favoreçam a atividade física e adoção de estilos de vida saudáveis.

Espera-se que os resultados apresentados subsidiem e fortaleçam ações e projetos contínuos de prevenção e combate ao sobrepeso e obesidade em estudantes universitários, e que aspectos voltados à autopercepção do estudante sejam uma temática trabalhada no ambiente universitário para valorizar a saúde mental e o protagonismo estudantil na escolha por hábitos saudáveis, dentro de um ambiente que estimula tais comportamentos. 
Este estudo teve algumas limitações. Utilizou-se o índice de massa corporal para verificação do sobrepeso e obesidade, método aceito para estudos em populações, contudo não permite a verificação da massa gorda e da massa livre de gordura dos estudantes.

\section{CONCLUSÃO}

Observou-se uma elevada prevalência de sobrepeso/obesidade e de insatisfação com a imagem corporal entre os universitários. Verificou-se associação da prevalência de sobrepeso com faixa etária, sexo, estado civil, trabalho, renda e consumo de carne vermelha; e associação entre a autopercepção da imagem corporal com autopercepção do estado de saúde, aptidão física e turno de estudo. Não houve associações entre o sobrepeso e as áreas dos cursos ou o tempo de vinculação com a universidade.

\section{AGRADECIMENTOS E CONFLITOS DE INTERESSE}

Agradecimento ao Centro Universitário INTA.

Não existem conflitos de interesse.

\section{CONTRIBUIÇÕES}

Michelle Alves Vasconcelos Ponte contribuiu com a elaboração e delineamento do estudo; aquisição, análise e interpretação de dados; redação e revisão do manuscrito; Sandra Celina Fernandes Fonseca, João José Saraiva da Fonseca, Maria Isabel Martins Mourão Carvalhal contribuíram com o delineamento do estudo; interpretação dos dados e revisão do manuscrito.

Manuscrito Baseado na tese de doutorado em Ciências da Educação da Universidade Trás-os-Montes e Alto Douro - UTAD/Portugal, Título: O processo de adaptação ao Ensino Superior: fatores associados ao rendimento acadêmico e a qualidade de vida em estudantes universitários, 2018, 176 páginas.

\section{REFERÊNCIAS}

1. Ministério da Saúde (BR). Guia alimentar para a população brasileira. $2^{\mathrm{a}}$. ed. Brasília: Ministério da Saúde; 2014.

2. Gardner RM, Stark K, Jackson NA, Friedman BN. Development and validation of two new scales for assessment of body image. Percept Mot Skills. 1999;89(3):981-93.

3. Brasil. Ministério da Saúde. Portaria $n^{\circ}$. 2.446/GM, de 11 de novembro de 2014. Redefine a Política Nacional de Promoção da Saúde (PNPS). Brasília: Ministério da Saúde; 2014.

4. World Health Organization. Obesity: preventing and managing the global epidemic. Genebra: World Health Organization; 2000.

5. Raynor HA, Champagne CM. Position of the Academy of Nutrition and Dietetics: interventions for the treatment of overweight and obesity in adults. J Acad Nutr Diet. 2016;116(1):129-47.

6. Instituto Brasileiro de Geografia e Estatística. Pesquisa nacional de saúde: 2013: ciclos de vida: Brasil e grandes regiões. Rio de Janeiro: IBGE; 2015.

7. Sousa TF, Fonseca AS, José HPM, Nahas MV. Validade e reprodutibilidade do questionário Indicadores de Saúde e Qualidade de Vida de Acadêmicos (Isaq-A). Arquivo de Ciências do Esporte. 2013;1(1):21-30.

8. Kakeshita IS, Almeida SS. Relação entre índice de massa corporal e a percepção da auto-imagem em universitários. Rev Saúde Pública [Internet]. 2006 [acesso em 2017 Abr 20];40(3):497-504. Disponível em: http://www.scielo.br/scielo.php?script=sci_arttext\&pid=S0034-89102006000300019\&lng=en

9. Brasil. Ministério da Saúde, Conselho Nacional de Saúde. Resolução nº. 466, de 12 de dezembro de 2012. Aprova as diretrizes e normas regulamentadoras da pesquisa envolvendo seres humanos. Brasília: Ministério da Saúde; 2012.

10. Silva SU, Barufaldi LA, Andrade SSCA, Santos MAS, Claro RM. Estado nutricional, imagem corporal e associação com comportamentos extremos para controle de peso em adolescentes brasileiros, Pesquisa 
Nacional de Saúde do Escolar de 2015. Rev Bras Epidemiol [Internet]. 2018 [acesso em 2018 Dez 29];21(Suppl 1):e180011. Disponível em: http://www.scielo.br/scielo.php?script=sci_arttext\&pid=S1415790X2018000200411\&lng=en

11. Alcântara da Silva P, Borrego R, Ferreira VS, Lavado E, Melo R, Rowland J, et al. Consumos e estilos de vida no ensino superior: o caso dos estudantes da ULisboa-2012. Lisboa: SICAD; 2015.

12. Ministério da Saúde (BR), Secretaria de Vigilância em Saúde. Vigitel Brasil 2017: vigilância de fatores de risco e proteção para doenças crônicas por inquérito telefônico: estimativas sobre frequência e distribuição sociodemográfica de fatores de risco e proteção para doenças crônicas nas capitais dos 26 estados brasileiros e no Distrito Federal em 2017. Brasília: Ministério da Saúde; 2018.

13. Sousa TF, Barbosa AR. Prevalências de excesso de peso corporal em universitários: análise de inquéritos repetidos. Rev Bras Epidemiol [Internet]. 2017 [acesso em 2018 Dez 12];20(4):586-97. Disponível em: http:// www.scielo.br/scielo.php?script=sci_arttext\&pid=S1415-790X2017000400586\&lng=en

14. Silva DAS, Quadros TMB, Gordia AP, Petroski EL. Associação do sobrepeso com variáveis sóciodemográficas e estilo de vida em universitários. Ciênc Saúde Coletiva [Internet]. 2011 [acesso em 2017 Mar 25]. Disponível em: http://www.scielo.br/scielo.php?script=sci_abstract\&pid=\$1413-81232011001200020\&Ing =pt\&nrm=iso\&tlng=pt

15. Vieira EES, Nobre RS, Ulbrich AZ, Carvalho GCN, Cortez RMA, Silva ARV. Sobrepeso e obesidade: associação com o nível socioeconômico de universitários. Rev Enferm UFPE online [Internet]. 2017 [acesso em 2018 Mar 18];11(10):3807-12. Disponível em: https://periodicos.ufpe.br/revistas/revistaenfermagem/ article/download/25195/24296

16. Lima CT, Ramos-Oliveira D, Barbosa C. Aspectos sociocognitivos da obesidade: estereótipos do excesso de peso. Psic Saúde \& Doenças [Internet]. 2017 [acesso em 2018 Dez 29];18(3):681-98. Disponível em: http:// www.scielo.mec.pt/scielo.php?script=sci_arttext\&pid=S1645-00862017000300005\&lng=pt. http://dx.doi. org/10.15309/17psd180305

17. Maciel V. Universidades terão até 10 milhões para prevenir e controlar a obesidade [Internet]. 2018 [acesso em 2018 Dez 10]. Disponível em: http://portalms.saude.gov.br/noticias/agencia-saude/44010-universidadesterao-ate-r-10-milhoes-para-prevenir-e-controlar-a-obesidade

18. Pires CGS, Mussi FC. Excesso de peso em universitários ingressantes e concluintes de um curso de Enfermagem. Esc Anna Nery [Internet]. 2016 [acesso em 2018 Dez 12];20(4):e20160098. Disponível em: http://www.scielo.br/pdf/ean/v20n4/1414-8145-ean-20-04-20160098.pdf

19. World Health Organization. Global health risks: Mortality and burden of disease attributable to selected major risks. Geneva: WHO; 2009.

20. Vieira CENK, Dantas DNA, Miranda LSMV, Araújo AKC, Monteiro Al, Enders BC. Programa de enfermagem saúde na escola: prevenção e controle de sobrepeso / obesidade em adolescentes. Rev Esc Enferm USP [Internet]. 2018 [acesso em 2018 Dez 11];52:e03339. Disponível em: http://www.scielo.br/scielo. php?script=sci_arttext\&pid=S0080-62342018000100433\&Ing=en. http://dx.doi.org/10.1590/s1980220x2017025403339

\section{Endereço para correspondência:}

Michelle Alves Vasconcelos Ponte

Rua Coronel Antônio Mendes Carneiro, 274

Bairro: Centro

CEP: 62010-160 - Sobral - CE - Brasil

E-mail: micc2005@hotmail.com

Como citar: Ponte MAV, Fonseca SCF, Carvalhal MIMM, da Fonseca JJS. Autoimagem corporal e prevalência de sobrepeso e obesidade em estudantes universitários. Rev Bras Promoç Saúde. 2019;32:8510. 